\title{
Barreras por las que la industria de cerveza artesanal no despega en el Perú
}

Eleazar Roberto QuisPe Orejón ${ }^{1}$ GUILlERMO QUINTANILla ALARCÓN ${ }^{2}$

\begin{abstract}
RESUMEN
La presente investigación se realizó con la finalidad de conocer las barreras que tienen los emprendedores de cerveza artesanal peruanos, que impide el incremento de su oferta de producción. Para conocer este impacto se aplicó un cuestionario y entrevistas a productores de cerveza artesanal de Lima Metropolitana y a consumidores de un segmento de mercado, encontrándose que la preferencia de los emprendedores de realizar promoción y publicidad en redes sociales es ineficiente. Un significativo porcentaje de emprendedores orienta sus productos a un amplio segmento de mercado. El sistema mixto de pago del impuesto selectivo al consumo es visto por el emprendedor como injusto y considerado un elemento que eleva el precio de sus productos. El consumidor aprecia el atributo sabor de la cerveza y muestra su disposición en pagar un precio alto.

Palabras-claves: Emprendedor; microcervecero; marketing y cerveza.
\end{abstract}

BARRIERS FOR WHICH THE CRAFT BEER INDUSTRY DOES NOT TAKE OFF IN PERU

\section{ABSTRACT}

This research was conducted in order to understand the barriers that Peruvian entrepreneurs craft beer, which prevents increasing their production supply. To meet this impact a questionnaire and interviews with producers of craft beer consumers in metropolitan Lima and a market segment was applied, being the preference of entrepreneurs to carry out promotion and advertising on social networks is inefficient. A significant percentage of entrepreneurs focuses its products to a broad market segment. The mixed system of payment of excise tax is seen as unfair by the entrepreneur and considered an element that raises the price of its products. The consumer appreciates the taste of beer attribute and shows its willingness to pay a high price.

Keywords: Entrepreneur; craft beer; brewers; marketing and beer.

\section{INTRODUCCIÓN}

En Perú los fabricantes de cerveza artesanal, a menudo llamados micro cervecerías, emprendedores, fabrican pequeñas cantidades de cerveza en varios sabores, grados de alcohol, colores y sólo venden en el mercado local. Los emprendedores peruanos enfrentan barreras que les impide el incremento de oferta de productos demandados por público objetivo. Estas dificultades que experimentan los productores de cerveza artesanal ocasionan que los consumidores no tengan acceso a un producto cervecero, producto que por la calidad de sus insumos es considerado un producto premium. La investigación de esta problemática se realizó por el interés de brindar a los emprendedores de cerveza artesanal peruanos, elementos de marketing que les permita identificar los impedimentos y entender la situación actual, consolidar su posición estratégica y ofertar sus productos a un nicho de mercado que aprecia el sabor y las características de un producto premium diferenciado si lo comparamos con un producto industrial.

Simonazzi (2009) afirma: "La cerveza es una bebida alcohólica elaborada por la fermentación de soluciones obtenidas de cereales y otros granos que contienen almidón" (p.5). Sobre el consumo de cerveza en Perú, Euromonitor International (2017) señala que el consumo per cápita de cerveza en Perú entre 2007 y 2016 ha experimentado un crecimiento de 32,4 litros hasta llegar a 46,90 litros por persona. Estas cifras corresponden al consumo de cerveza industrial. En Perú, aunque no se evidencia tradición de fabricantes cerveceros, la tendencia mundial es la aparición de micro cervecerías o cerveceras artesanales, esto viene ocurriendo en Perú, aunque de una manera aún incipiente.

Los emprendedores de cerveza artesanal están identificados como MIPYME ${ }^{3}$, tienen una contribución importante en la industria y en el empleo nacional, en ese sentido, la OEE ${ }^{4}$ (2017) sostiene que este segmento empresarial representa el $99.5 \%$ del total de empresas formales en la economía peruana el $94.9 \%$ son microempresas, $4.5 \%$ pequeña y $0.2 \%$ mediana

1 Ingeniero Industrial. Email: robertoquispeo@gmail.com

2 Magister en Gestión de Políticas Públicas. Docente de la Universidad Nacional del Callao. Email: gquintal@unac.pe

3 MIPYME:Micro, pequeña y mediana empres

4 OEE: Oficina de Estudios Económicos del Ministerio de la Producción 


\section{METODOLOGIA}

La investigación propuesta es de tipo descriptiva porque pretende describir las características de los emprendedores de cerveza artesanal y de los consumidores, y explicar las barreras por las que la industria de cerveza artesanal no despega en el Perú. En ese sentido Grande y Abascal (2009) sostienen que las investigaciones descriptivas, explican situaciones y pueden perseguir muchos objetivos, entre ellos describir las características de grupos, de consumidores, empresas, marcas o intermediarios; encontrar fortalezas y debilidades de empresas, productos; entre otras características de este tipo de investigación. Se emplea también la investigación de tipo explicativa, Yuni y Urbano (2006) afirman que en este tipo de investigación se intenta examinar la naturaleza de las relaciones, la causa y eficacia de una o más variables mediante comparaciones de variables; en ese sentido se examinarán las razones por la cuales los emprendedores de cerveza artesanal peruanos ofertan sus productos muy discretamente si lo comparamos con los países de la región.

Las barreras de ingreso al mercado peruano de cerveza artesanal tienen relación con las variables producto ofertado, clientes y con la variable ofertantes o emprendedores de cerveza artesanal.

Debido a la naturaleza descriptiva del estudio, se realizó la investigación usando fuentes de datos primarias. La fase inicial exploratoria de tipo cualitativa orientada a los consumidores de cerveza se realizó con la técnica de focus group durante el mes de enero 2017, según Flores (2013) esta técnica resulta fructífera para explorar las percepciones, sentimientos y pensamientos de los actores sociales sobre determinados temas. Kotler y Armstrong (2013) sostienen que: "La investigación por encuestas, el método más ampliamente utilizado para la recopilación de datos primarios, es el método mejor estudiado para la recopilación de información descriptiva" (p. 106). Aznar, Gallego y Medianero (2015) sostienen que: "Un cuestionario es un documento que contiene una serie de preguntas diseñadas con la finalidad de generar datos necesarios para la consecución de los objetivos marcados por el proyecto de investigación" (p. 65). Malhotra (2008) manifiesta que la encuesta y observación son las dos principales técnicas para obtener datos cuantitativos primarios en la investigación descriptiva y afirma que "el tipo de información obtenida en un cuestionario puede clasificarse como: 1. información básica, 2. información de clasificación y 3. información de identificación" (p. 315). Por lo antes citado se reconoce al cuestionario como fuente primaria valida y confiable en la investigación de mercados.

Entonces se identifica el segmento de mercado de posibles consumidores de cerveza artesanal y luego se calcula el tamaño de la muestra poblacional a quienes se aplicó el cuestionario el mes de febrero 2017. El cuestionario para el consumidor tiene tres bloques de preguntas. Un primer bloque incluye preguntas para identificar indicadores demográficos de sexo, edad y distrito de residencia. El segundo bloque es para obtener indicadores referentes al consumo de alcohol y cerveza. El tercer bloque de preguntas es referente al consumo de cerveza artesanal e indicadores como frecuencia de consumo, canal de compra, recordación de marca, medio de información y atributos o características valorizados en su consumo. El proceso de levantamiento de información se desarrolló los meses de febrero y marzo 2017, para consumidores de cerveza aplicando el cuestionario a una muestra conformada por hombres y mujeres de 25 a 45 años de edad, de nivel socioeconómico $\mathrm{A}, \mathrm{B}$ de los distritos de $\mathrm{Mi}$ raflores, San Isidro, San Borja, Santiago de Surco y la Molina correspondientes a Lima Metropolitana, el tipo de cuestionario ha sido directo, y el muestreo de tipo aleatorio simple.

El instrumento de medición usado para obtener información cuantitativa de los emprendedores de cerveza artesanal consiste en un cuestionario formado por tres bloques de preguntas. El primer bloque incluye preguntas sobre el producto ofertado por el emprendedor, para obtener indicadores de procedencia de la materia prima, e indicadores de inversión en promoción y publicidad del producto. El segundo bloque de preguntas es para conocer el segmento de mercado que atiende el emprendedor, canal de distribución, el tercer bloque de preguntas es para obtener información del emprendedor sobre aspectos tributarios en el sector industrial. El levantamiento de información para los emprendedores de cerveza artesanal se desarrolló aplicando un cuestionario bajo la técnica de entrevista directa, a emprendedores de cerveza artesanal ubicados en Lima Metropolitana, bajo un tipo de muestreo aleatorio simple.

\section{RESULTADOS}

\section{Promoción}

Kotler y Armstrong (2013) sostienen que: "La mezcla de promoción consiste en la mezcla específica de publicidad, relaciones públicas, ventas personales promoción de ventas y herramienta de marke- 
ting directo que utiliza la compañía para comunicar valor para el cliente de forma persuasiva y establecer relaciones con éste" (p.408). Los emprendedores de cerveza artesanal entrevistados un $53.8 \%$ destinan hasta S/ 500 soles mensuales en realizar promoción y publicidad. Un $7.79 \%$ menciona invertir casi nada, no precisa la cantidad invertida y un emprendedor menciona no invertir ya que desde su punto de vista I cliente llega solo. Los niveles de inversión identificados son presentados en la Tabla 1.

Tabla 1. Emprendedor de cerveza artesanal, Inversión en publicidad

\begin{tabular}{|l|c|c|c|}
\hline Inversión en Soles & Frecuencia & Porcentaje & $\begin{array}{c}\text { Porcentaje } \\
\text { acumulado }\end{array}$ \\
\hline Casi nada & 2 & $7.7 \%$ & $7.7 \%$ \\
\hline No precisa & 4 & $15.4 \%$ & $23.1 \%$ \\
\hline No invierte & 1 & $3.8 \%$ & $26.9 \%$ \\
\hline 1 a 500 & 7 & $26.9 \%$ & $53.8 \%$ \\
\hline 501 a 1.000 & 3 & $11.5 \%$ & $65.3 \%$ \\
\hline 1.001 a 2.000 & 2 & $7.0 \%$ & $72.3 \%$ \\
\hline 2.001 a 3.000 & 3 & $11.5 \%$ & $83.8 \%$ \\
\hline 3.001 a 4.000 & 3 & $11.5 \%$ & $95.3 \%$ \\
\hline 4.001 a 5.000 & 1 & $3.8 \%$ & $100.0 \%$ \\
\hline Total & $\mathbf{2 6}$ & $\mathbf{1 0 0 . 0 \%}$ & \\
\hline
\end{tabular}

Fuente: Elaboración propia
La Figura 1 muestra que un $80 \%$ de los emprendedores de cerveza artesanal prefieren realizar la promoción y publicidad usando la red social Facebook, no precisando como hacerlo y promocionando boca a boca la oferta de sus productos.

\section{Materia Prima}

Sobre la materia prima para la elaboración de cerveza artesanal Reyes (2005) señala que básicamente, los ingredientes son la cebada, el lúpulo, la levadura y el agua, mezclados y pasados por un proceso de fermentación, maduración y pasteurización dan como producto un líquido ligero, brillante y efervescente, de sabor fresco y suavemente amargo. El $100 \%$ de la muestra de emprendedores de cerveza artesanal peruanos mencionan usar materia prima importada principalmente de: Bélgica, Alemania, Estados Unidos, Suecia, México, Chile y Brasil principalmente.

\section{Segmentación}

Es el proceso por medio del cual el mercado total de un producto o servicio es dividido en grupos relativamente homogéneos atendiendo a sus características y necesidades particulares (Baena, 2011). En la muestra entrevistada de 362 consumidores, 247 correspondieron a consumidores de

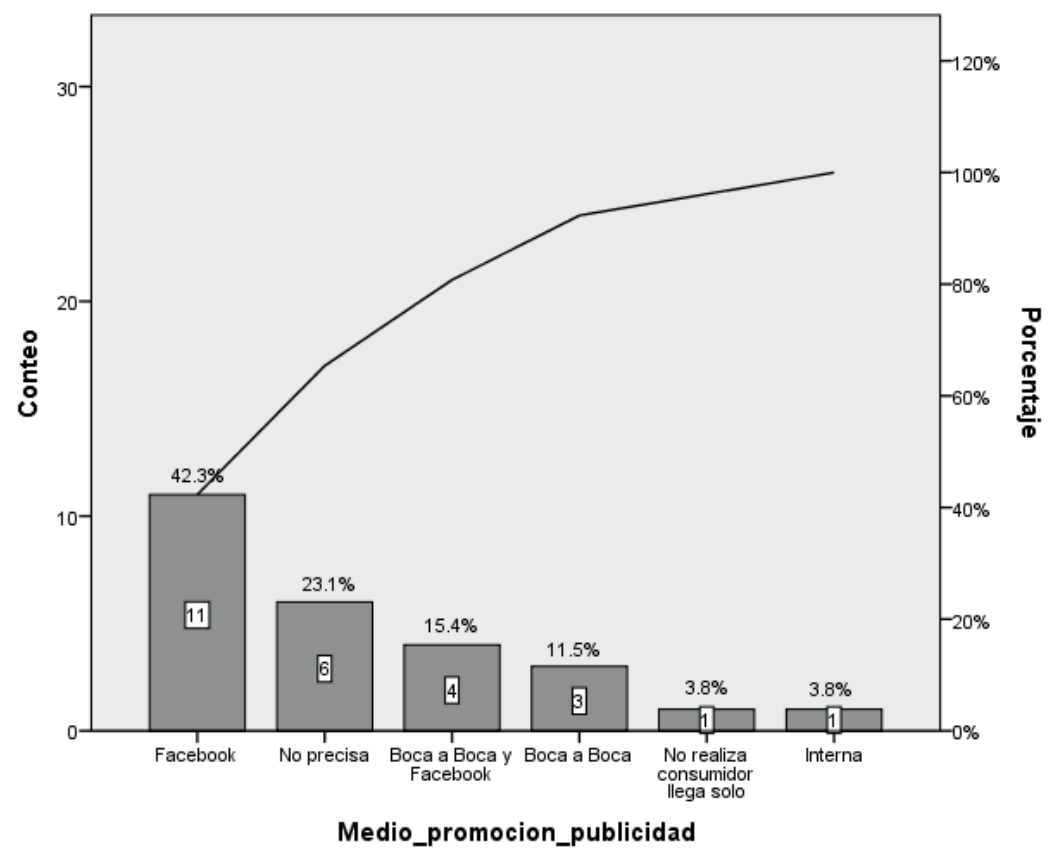

Figura 1. Preferencia en publicidad del emprendedor de cerveza artesanal

Fuente: Elaboración propia, software SPSS. 
sexo masculino, un $56.7 \%$ de los consumidores masculinos (140) mencionaron haber consumido al menos una vez la cerveza artesanal y un $43.3 \%$ mencionó desconocer el producto (107). Respecto a consumidores de sexo femenino en la misma muestra se entrevistó a 115 consumidores, se encontró que un $52.1 \%$ de consumidores femeninos (60) mencionaron haber consumido al menos una vez la cerveza artesanal y un $47.9 \%$ mencionó desconocer el producto (55). Para analizar en la muestra la relación de dependencia de la segmentación demográfica del sexo y el consumo de cerveza artesanal están relacionadas se parte de las siguientes hipótesis:

$H_{0}=$ Las variables demográfica sexo y consumo de cerveza artesanal son independientes

$\mathrm{H}_{1}=$ Las variables demográfica sexo y consumo de cerveza artesanal están relacionadas

La prueba Chi-cuadrado mostrada en la Tabla 2, presenta el resultado de significación asintótica (bilateral) de 0.422 cifra que es mayor al grado de error de 0.05; según el cual se acepta la hipótesis nula de independencia y se concluye que la varia- ble demográfica sexo y consumo de cerveza son independientes.

En referencia a la segmentación encontrada por edad, en la base de datos: entrevistas-consumidores cerveza artesanal.sav se ha creó la variable dicotómica Consumo_cerveza_artesanal $=\left\{\begin{array}{ll}0.1 & 1\end{array}\right.$, donde $0=$ no consume y $1=$ si consume; la variable dicotómica se usó para filtrar la base de datos y obtener consumidores por edad (ver Tabla 3 ). La muestra en estudio, evidencia que de los 200 consumidores que mencionaron haber consumido cerveza artesanal al menos una vez la edad preferida de los consumidores peruanos corresponde a la edad de 26 a 30 años, para ambos géneros; masculino $42.9 \%$ y femenino $46.7 \%$.

La dependencia de la segmentación demográfica por edad del consumidor con el consumo de cerveza artesanal se realiza con la siguiente hipótesis:

Ho = Las variables demográfica edad sexo y el consumo de cerveza artesanal son independientes

H1 = Las variables demográfica edad sexo y consumo de cerveza artesanal están relacionadas.

Tabla 2. Chi-cuadrado, variable demográfica sexo y consumo de cerveza artesanal

\begin{tabular}{|l|c|c|c|c|c|}
\cline { 2 - 5 } \multicolumn{1}{c|}{} & Valor & gl & $\begin{array}{c}\text { Significación } \\
\text { asintótica } \\
\text { (bilateral) }\end{array}$ & $\begin{array}{c}\text { Significación } \\
\text { exacta } \\
\text { (bilateral) }\end{array}$ & $\begin{array}{c}\text { Significación } \\
\text { Exacta } \\
\text { (unilateral) }\end{array}$ \\
\hline Chi-cuadrado de Pearson & $.644(\mathrm{a})$ & 1 & .422 & & \\
\hline Corrección de continuidad (b) & .475 & 1 & .491 & & \\
\hline Razón de verosimilitud & .643 & 1 & .423 & & .429 \\
\hline Prueba exacta de Fisher & & & & & .423 \\
\hline Asociación lineal por lineal & .643 & 1 & & & \\
\hline N de casos válidos & 362 & & & & \\
\hline
\end{tabular}

Fuente: Elaboración propia, software SPSS.

Tabla 3. Consumo de cerveza artesanal por edad

\begin{tabular}{|l|l|c|c|c|c|c|}
\cline { 3 - 6 } \multicolumn{2}{l|}{} & \multicolumn{4}{c|}{ Edad } & \multicolumn{2}{c|}{} \\
\hline Género & Recuento & $\mathbf{2 6 - 3 0}$ & $\mathbf{3 1 - 3 5}$ & $\mathbf{3 6 - 4 0}$ & $\mathbf{4 1 - 4 5}$ & Tamaño muestra \\
\hline Masculino & Consumidores & 60 & 39 & 22 & 19 & 140 \\
\hline & $\%$ dentro de género & $42.9 \%$ & $27.9 \%$ & $15.7 \%$ & $13.6 \%$ & $100.0 \%$ \\
\hline Femenino & Consumidores & 28 & 11 & 11 & 10 & 60 \\
\hline & $\%$ dentro del género & $46.7 \%$ & $18.3 \%$ & $18.3 \%$ & $16.7 \%$ & $100.0 \%$ \\
\hline
\end{tabular}

Fuente: Elaboración propia, software SPSS. 
La prueba Chi-cuadrado mostrada en la Tabla 4 , presenta el resultado de significación asintótica (bilateral) de 0.549 cifra que es mayor al grado de error de 0.05; según el cual se acepta la hipótesis nula de independencia y se concluye que la variable demográfica edad y consumo de cerveza artesanal son independientes.

Se observa una preferencia $(73.1 \%)$ de los emprendedores de cerveza artesanal en ofertar sus productos al $\mathrm{NSE}^{5} \mathrm{~A}, \mathrm{~B}$, incluyendo extranjeros y se evidencia que un $19.2 \%$ de emprendedores orientan sus productos a todos los NSE, ver Tabla 5 . Al formular la pregunta para conocer la edad del consumidor que atiende el emprendedor, se encuentra que un $61.5 \%$ de emprendedores de cerveza artesanal no precisa la edad de sus consumidores y el porcentaje restante evidencia una gran dispersión en la edad de sus consumidores.

\section{Plaza}

Un $92.3 \%$ de los emprendedores de cerveza artesanal entrevistados manifestaron ofertar sus pro- ductos en los restaurants, $73.1 \%$ mencionan ofertar sus productos en los supermercados y en licorerías $53.8 \%$. Cuando se habla de distribución se está hablando de dos elementos la distribución física y los canales de Distribución. Son conceptos que van juntos, aunque se traten por separado (Giraldo y David, 2016)

Luego de aplicar la encuesta a los consumidores y se observa que los consumidores prefieren comprar el producto en Pub/bar (47\%), restaurant $(20 \%)$ y supermercado (16\%), lo que hace un $83 \%$ de la preferencia del consumidor. Se evidencia una contradicción con lo obtenido en la entrevista a los emprendedores de cerveza artesanal y los consumidores. Los emprendedores mencionan su principal preferencia de ofertar sus productos en el restaurant $(92.3 \%)$, supermercado $(73.1 \%)$; mientras que la muestra de los consumidores evidencia que solo en un $20 \%$ consumen la cerveza en el restaurant, ver Figura 2; el consumidor prefiere (47\%) consumir la cerveza artesanal en el pub/bar.

Tabla 4. Chi-Cuadrado, variable demográfica edad y consumo de cerveza artesanal

\begin{tabular}{|l|c|c|c|}
\cline { 2 - 4 } \multicolumn{1}{c|}{} & Valor & gl & Significación asintótica (bilateral) \\
\hline Chi-cuadrado de Pearson & $2.144(\mathrm{a})$ & 3 & .549 \\
\hline Razón de verosimilitud & 2.196 & 3 & .523 \\
\hline N de casos válidos & 200 & & \\
\hline
\end{tabular}

Nota. (a) 0 casillas ( $0 \%$ ) han esperado un recuento menor que 5.

El recuento mínimo esperado es 8.70 .

Fuente: Elaboración propia, software SPSS.

Tabla 5. Consumo de cerveza artesanal por segmento

\begin{tabular}{|l|c|c|c|}
\hline \multicolumn{1}{|c|}{ Segmento } & Frecuencia & Porcentaje & Porcentaje Acumulado \\
\hline A & 4 & $15.4 \%$ & $15.4 \%$ \\
\hline A, B & 13 & $50.0 \%$ & $65.4 \%$ \\
\hline A, B y extranjeros & 1 & $3.8 \%$ & $69.2 \%$ \\
\hline A, B y extranjeros, personas que pueden pagar precio. & 1 & $3.8 \%$ & $73.1 \%$ \\
\hline A, B, C & 1 & $3.8 \%$ & $76.9 \%$ \\
\hline B cono norte & 1 & $3.8 \%$ & $80.8 \%$ \\
\hline Extranjeros & 1 & $3.8 \%$ & $84.6 \%$ \\
\hline Todos & 4 & $15.4 \%$ & $100.0 \%$ \\
\hline Total & $\mathbf{2 6}$ & $\mathbf{1 0 0 . 0 \%}$ & \\
\hline
\end{tabular}

Fuente: Elaboración propia, software SPSS. 


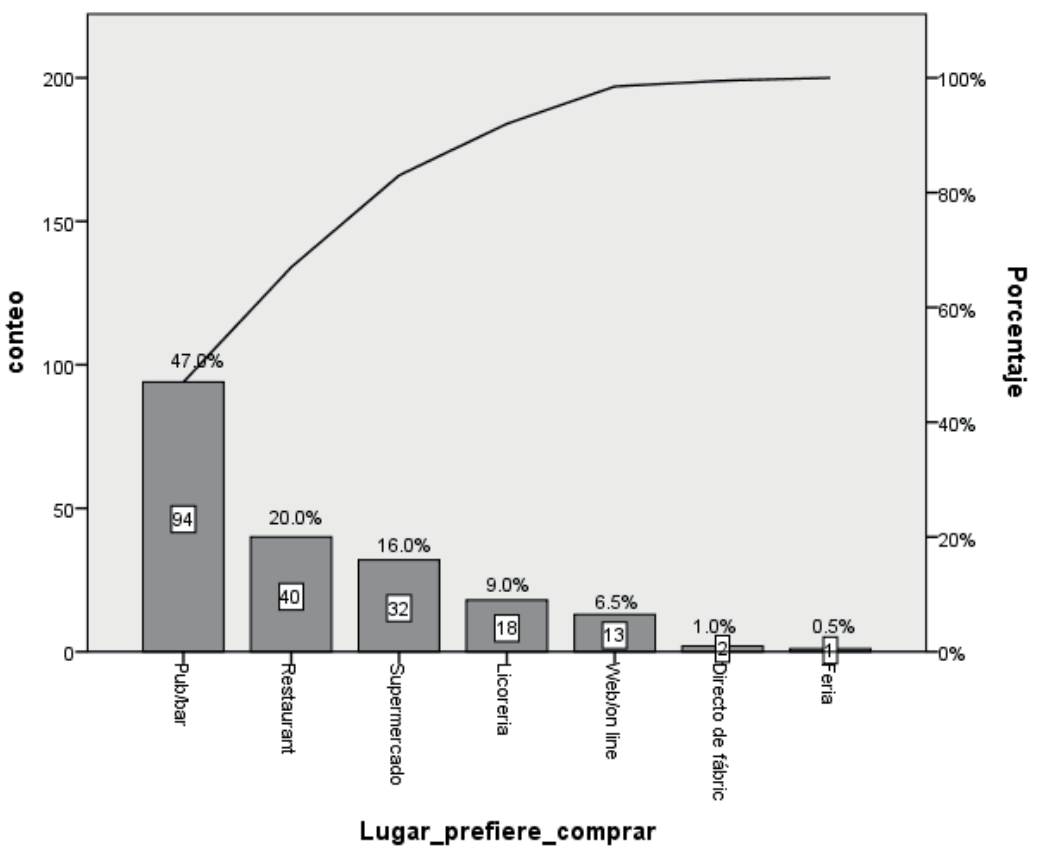

Figura 2. Diagrama de Pareto. Consumidor preferencia de compra.

Fuente. Elaboración propia, software SPSS.

Si al consumidor se le pregunta ¿Cuál es la cerveza que prefiere?, la respuesta es inmediata y evidencia una preferencia por la cerveza industrial, 60.63\% prefieren Pilsen Callao, 22.54 Cuzqueña, $7.30 \%$ Cristal y luego otras marcas como Corona y Brahma. En la muestra las tres marcas industriales tienen la preferencia del consumidor $(90.47 \%)$.

Respecto a la cerveza artesanal, cuando a la muestra se le formula la pregunta ¿cuál es la cerveza que recuerda haber consumido?, un $50 \%$ de los entrevistados manifiesta no recordar la marca, 19\% recuerda la cerveza Barbarian, 8.5\% Candelaria, $4.5 \%$ haber consumido Barranco Beer Company y Nuevo Mundo 4\%, el resto de marcas tiene una recordación menor.

\section{Atributo valorado en la cerveza artesanal}

La muestra estadística presenta 200 consumidores de cerveza artesanal, 140 corresponden a personas de sexo masculino y 60 personas de sexo femenino. En ambos segmentos el principal atributo valorado en la cerveza artesanal es el 'sabor' y en segundo lugar el grado de alcohol (17.1\%) para el género masculino y el femenino prefiere en segundo lugar los ingredientes (11.7\%) ver Tabla 6.

Esta investigación analiza la segmentación demográfica de sexo con los atributos de la cerveza artesanal de: alcohol, calidad de los ingredientes, precio y sabor de los consumidores. En la base de datos: entrevistas-consumidores cerveza artesanal. sav se ha creado la variable dicotómica Consumo_

Tabla 6. Atributo valorado en cerveza artesanal

\begin{tabular}{|l|c|c|c|c|c|}
\cline { 2 - 5 } \multicolumn{1}{c|}{} & \multicolumn{5}{c|}{ Atributo } \\
\hline Género & Grado alcohol & Ingredientes & Precio & Sabor & Total \\
\hline Masculino & 24 & 19 & 2 & 95 & 140 \\
\hline$\%$ dentro del género & $17.1 \%$ & $13.6 \%$ & $1.4 \%$ & $67.9 \%$ & \\
\hline Femenino & 6 & 7 & 2 & 45 & 60 \\
\hline$\%$ dentro del género & $10.0 \%$ & $11.7 \%$ & $3.3 \%$ & $75.0 \%$ & \\
\hline
\end{tabular}

Fuente: Elaboración propia, software SPSS. 
cerveza_artesanal $=\{0.1\}$, donde $0=$ no consume y 1 = si consume; la variable dicotómica será usada para filtrar la base de datos. La dependencia de la segmentación demográfica sexo con los atributos de la cerveza artesanal de: alcohol, calidad de los ingredientes, precio y sabor de los consumidores; se realiza con la siguiente hipótesis:

Ho = Las variables demográfica sexo y los atributos de la cerveza artesanal de: alcohol, calidad de los ingredientes, precio y sabor son independientes

H1 = Las variables demográfica sexo y los atributos de la cerveza artesanal de: alcohol, calidad de los ingredientes, precio y sabor están relacionadas.

La prueba Chi-cuadrado mostrada en la Tabla 7 presenta el resultado de significación asintótica (bilateral) de 0.455 cifra que es mayor al grado de error de 0.05; según el cual se acepta la hipótesis nula de independencia y se concluye que la variable demográfica género y los atributos de la cerveza artesanal de: alcohol, calidad de los ingredientes, precio y sabor son independientes.

Tabla 7. Chi-Cuadrado, variable género y consumo de cerveza artesanal

\begin{tabular}{|l|c|c|c|}
\cline { 2 - 4 } \multicolumn{1}{c|}{} & Valor & gl & $\begin{array}{c}\text { Significación } \\
\text { asintótica } \\
\text { (bilateral) }\end{array}$ \\
\hline Chi-cuadrado de Pearson & $2.614(\mathrm{a})$ & 3 & .455 \\
\hline Razón de verosimilitud & 2.663 & 3 & .447 \\
\hline N de casos válidos & 200 & & \\
\hline
\end{tabular}

Fuente: Elaboración propia, software SPSS.

\section{Precio}

El diseño de una política de precios es muy complejo, pero siempre se debe tener claro que un precio es la expresión de un valor (Villanueva y De Toro, 2017). Se encuentra en la muestra de los consumidores de Lima metropolitana que un $44.5 \%$ compran la cerveza artesanal entre S/ 11 a S/15 soles, un $26 \%$ entre $S / 6$ a S/ 10 y un $25 \%$ entre $S / 16$ a $\mathrm{S} / 20$, lo que significa que el $95 \%$ de la muestra está dispuesta a pagar por una cerveza artesanal de 300 cc el importe entre S/ 6 y S/ 20. Apenas un $4.5 \%$ de los consumidores manifiesta haber consumido una cerveza de S/ 21 a S/ 30. (ver Figura 3)

Se observa que un $4.5 \%$ de la muestra de consumidores de cerveza artesanal han manifestado su disposición por consumir una cerveza de S/ 21 a S/ 30. lo que puede significar que han consumido una cerveza artesanal de $300 \mathrm{cc}$ importada de Bélgica o Alemania, ya que en el mercado local no hay productores nacionales que oferten la cerveza a ese precio.

\section{Impuesto selectivo al consumo (ISC)}

En Perú desde el 2013 los cerveceros artesanales pagan los impuestos de acuerdo con un sistema mixto, en este sistema si la bebida alcohólica tiene de 0 a 6 grados de alcohol debe pagar S/. 1.25 por cada litro producido o el $30 \%$ del precio de venta al público, y si tiene de 6 a 20 grados de alcohol debe pagar S/ 2.50 por cada litro producido o el $25 \%$ del precio de venta al público, finalmente si la bebida alcohólica tiene más de 20 grados de alcohol debe pagar S/ 3.40 por cada litro producido o el $25 \%$ del precio de venta al público. En este sistema mixto

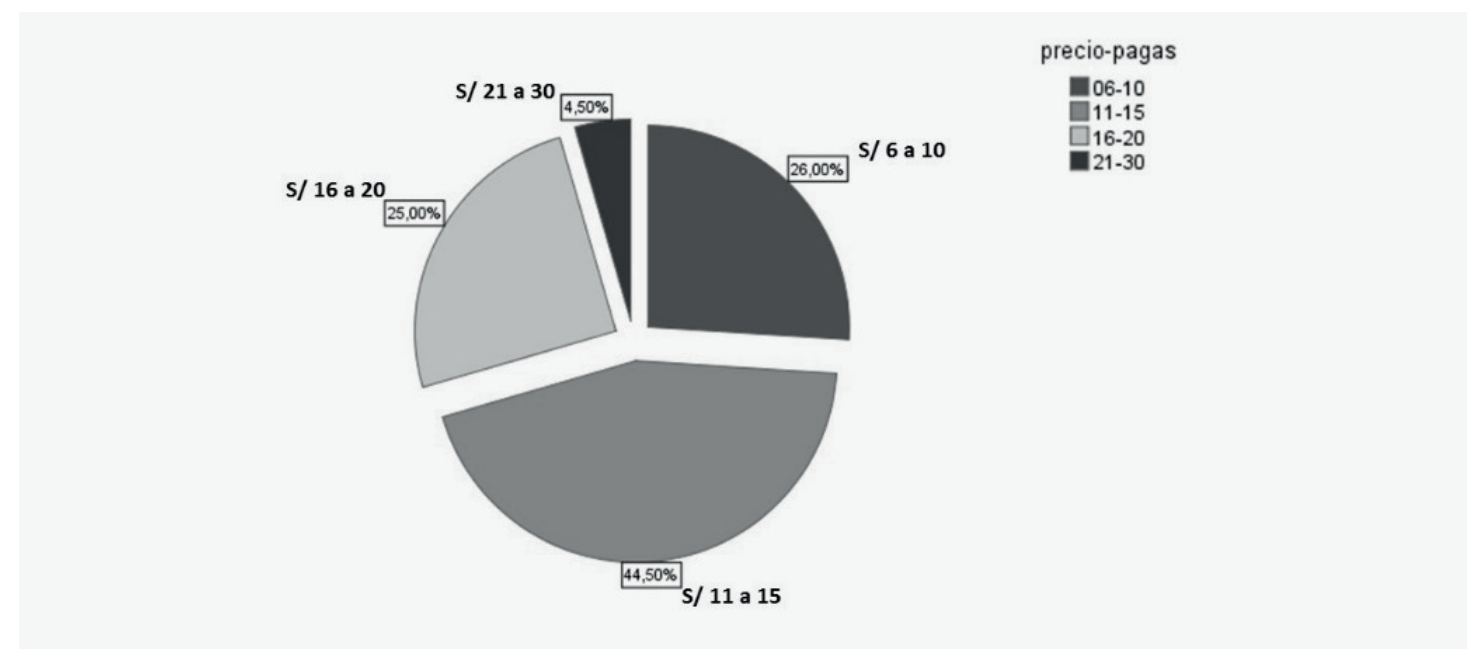

Figura 3. Precio pagado por consumidor de Lima Metropolitana

Fuente. Elaboración propia, software SPSS. 
el emprendedor de cerveza artesanal debe pagar de acuerdo con la ley, siempre el mayor de los montos que resulta de realizar el cálculo en cada uno de los esquemas antes descritos. Los cerveceros artesanales en su totalidad afirman que el ISC y el sistema mixto no los favorece, que este es un impuesto es elevado e injusto y que debe aplicarse a los litros producidos. Apenas un emprendedor manifestó su entusiasmo al afirmar que el sistema mixto actual no lo afecta porque ingresó al mercado con ese impuesto y aún se mantiene. Se puede identificar que los emprendedores de cerveza artesanal peruanos encuentran el ISC y el sistema mixto como desfavorable.

\section{DISCUSIÓN}

Existen diferentes estrategias genéricas David (2013) sostiene que: "De acuerdo con Porter, las estrategias permiten que las organizaciones obtengan una ventaja competitiva a partir de tres ejes fundamentales: liderazgo en costos, diferenciación y enfoque" (p. 148).

\section{Análisis de la industria: La matriz de evaluación de factores externos (EFE)}

El ambiente general es el más amplio, Palacios (2009) menciona que: "Funciona como un sistema general que faceta a todos sus componentes e integrantes de modo genérico. Este ambiente de variables externas es el escenario más amplio en donde ocurren todos los fenómenos que influyen en las organizaciones" (p. 19).

La matriz de factores externos (EFE) ver Tabla 8, será usada en esta investigación para resumir y evaluar económica, social y cultural, demográfica, ambiental, política, legal, gubernamental, tecnológica del sector industrial de cerveceros artesanales. La cerveza artesanal se está haciendo popular en Perú, y se distingue de la cerveza normal debido a que tales productos se elaboran por pequeñas em-

Tabla 8. Matriz de factores externos

\begin{tabular}{|c|c|c|c|}
\hline Factores de éxito & Peso & Calificación & Ponderado \\
\hline \multicolumn{4}{|l|}{ Oportunidades } \\
\hline Aumento de confianza en consumidores con respecto a la economía peruana. & 0.10 & 3 & 0.30 \\
\hline Sin productos sustitutos. & 0.10 & 2 & 0.20 \\
\hline Oferta de cervezas saborizadas. & 0.10 & 2 & 0.20 \\
\hline En Perú no hay cervezas sin alcohol o con bajos grados de alcohol. & 0.06 & 1 & 0.06 \\
\hline Conquistar segmento de mujeres (no solo con opciones más ligeras o menos amargas). & 0.06 & 1 & 0.06 \\
\hline Cultura gastronómica peruana. & 0.05 & 1 & 0.05 \\
\hline \% de población mayor a 35 años que puede ser considerada como consumidores & 0.03 & 2 & 0.06 \\
\hline \multicolumn{4}{|l|}{ Amenazas } \\
\hline Productores industriales con alto volumen y bajo precio. & 0.05 & 1 & 0.05 \\
\hline Dependencia de materia prima importada. & 0.10 & 2 & 0.20 \\
\hline Ser considerado producto exclusivo y para gente adinerada. & 0.05 & 3 & 0.15 \\
\hline Impuestos y sistema mixto desfavorable. & 0.10 & 3 & 0.30 \\
\hline Posible ingreso de competidores extranjeros. & 0.20 & 1 & 0.20 \\
\hline $\begin{array}{l}\text { En Perú la cerveza está asociada mayormente a celebraciones, actividades festivas (en } \\
\text { provincias la contribución es en caja). }\end{array}$ & 0.05 & 1 & 0.05 \\
\hline Sin economía de escala. & 0.05 & 2 & 0.10 \\
\hline Tecnología no industrial. & 0.05 & 2 & 0.10 \\
\hline Gremio que los agrupa escasa actividad en fomento, publicidad y promoción. & 0.02 & 1 & 0.02 \\
\hline Sin participación en October Fest. & 0.01 & 1 & 0.01 \\
\hline Total & 1.00 & & 1.93 \\
\hline
\end{tabular}

Fuente. Elaboración propia. 
presas de una forma no industrial. Con bajos volúmenes de producción, las cervezas artesanales se ofrecen a precios altos, y por lo tanto se dirigen a un segmento de consumidores con ingresos altos, a nichos dispuestos a pagar más por esta cerveza que las pueden encontrar incluso en restaurantes de lujo. (Euromonitor International, 2017). La explicación del aumento de la confianza de los consumidores respecto a la economía peruana se debe a que de acuerdo al INEI ${ }^{6}$, entre el 2001 y 2016 el PBI por habitante creció a una tasa anual de 3.5\%, pasando de 13 mil 634 soles en el año 2011 a 15 mil 914 soles en el año 2016, este es el valor más alto desde 1950 .

La Tabla 8 muestra un total ponderado de 1.93; esto indica que los emprendedores de cerveza artesanal peruanos están justo por debajo de la media en su esfuerzo por conseguir estrategias que capitalicen oportunidades externas y eviten las amenazas. Tiene más peso las amenazas que las oportunidades.

\section{Análisis de la industria: La matriz de evaluación de factores internos (EFI)}

Fernández (2004) menciona que "Luego puede pasarse al análisis interno, con el inventario, jerarquización, selección y análisis de los temas estratégicos" (p. 43).
La matriz de factores externos (EFI) será usada en esta investigación para evaluar las fortalezas y debilidades principales en las áreas funcionales. La Tabla 9 muestra un total ponderado de 2.25, esta cifra está justo por sobre la media, lo que significa que los emprendedores de cerveza artesanal peruanos realizan estrategias que capitalizan las fortalezas internas y neutralizan las debilidades. Esto significa que tiene más peso las fortalezas que las debilidades.

\section{Análisis FODA}

En esta investigación y luego de analizar matriz de factores externos (EFE) se identifica que los emprendedores de cerveza artesanal peruanos experimentan un mayor peso o influencia de las amenazas externas a su actividad productiva. El análisis de la matriz de actores internos (EFI) identifica que los emprendedores de cerveza artesanal peruanos hacen esfuerzos por aprovechar sus fortalezas internas y de esta forma disminuir o atenuar sus debilidades internas.

Con las matrices EFE y EFI se desarrolla en análisis FODA en este análisis se identifica que los emprendedores de cerveza artesanal peruanos se encuentran en una posición ofensiva.

Tabla 9. Matriz de factores internos

\begin{tabular}{|c|c|c|c|}
\hline Factores de éxito & Peso & Calificación & Ponderado \\
\hline \multicolumn{4}{|l|}{ Fortalezas } \\
\hline Consumidor aprecia sabor diferente. & 0.10 & 4 & 0.40 \\
\hline Entusiasmo por emprendimiento e innovación. & 0.05 & 4 & 0.20 \\
\hline Pocos trabajadores. & 0.08 & 3 & 0.30 \\
\hline Producto premium. & 0.10 & 3 & 0.30 \\
\hline Calidad de insumos. & 0.10 & 4 & 0.40 \\
\hline Maridaje con comida peruana. & 0.40 & 3 & 0.12 \\
\hline \multicolumn{4}{|l|}{ Debilidades } \\
\hline Segmentación de mercado. & 0.10 & 1 & 0.10 \\
\hline Distribución. & 0.05 & 1 & 0.05 \\
\hline Precio alto. & 0.05 & 1 & 0.05 \\
\hline Baja capacidad de producción & 0.06 & 2 & 0.12 \\
\hline Baja inversión & 0.07 & 1 & 0.07 \\
\hline Recordación de marca & 0.10 & 1 & 0.10 \\
\hline \multicolumn{4}{|l|}{ Sin economía de escala. } \\
\hline Uso tecnología BTL para promoción y publicidad & 0.10 & 1 & 0.10 \\
\hline Total & 1.00 & & 2.25 \\
\hline
\end{tabular}

Fuente. Elaboración propia. 


\section{CONCLUSIONES}

Los impedimentos que experimentan los cerveceros artesanales peruanos para ocupar nichos de mercado e incrementar la oferta de productos son los siguientes:

El uso de las redes sociales de los emprendedores peruanos como instrumento para realizar promoción y publicidad no logra el impacto deseado ya que solo un $20 \%$ de personas usa estos medios para informarse de productos.

Los productores de cerveza artesanal peruanos mayormente orientan este producto al segmento $A$ y $\mathrm{B}$ y un $15 \%$ de los productores menciona orientar el producto a todos los NSE, esto diluye sus esfuerzos, entonces debería realizarse una alta segmentación para identificar nichos de mercado.

El impuesto selectivo al consumo de cerveza artesanal se calcula bajo un sistema mixto en función del grado de alcohol y del litro producido de cerveza.

Esta investigación ha evidenciado en el consumidor peruano lo siguiente:

El atributo más valorado por el consumidor peruano de cerveza artesanal es el sabor diferente $(70 \%$ de los encuestados), le sigue el grado de alcohol 15\% de los encuestados) y calidad de los ingredientes con un $13 \%$. Esto significa que el atributo 'sabor' debería ser usado como una característica de diferenciación del producto.

Un $45 \%$ de los encuestados puede pagar un precio de S/ 11 a S/ 15 soles por una cerveza artesanal de $300 \mathrm{cc}$

El estudio realizado ha confirmado la dependencia de la materia prima de proveedores extranjeros.

El análisis FODA determina que los productores de cerveza artesanal peruanos pueden optar una posición ofensiva, podrían aprovechar todas sus fortalezas para atenuar las amenazas externas en el sector industrial.

\section{REFERENCIAS BIBLIOGRAFICAS}

[1] Aznar, J., Gallego, R., y Medianero, M. (Eds.). (2015). Investigación y recogida de información de mercados (uf1780), Madrid: Editorial CEP S.I. Recuperado de http://ebookcentral. proquest.com
[2] Baena, V. (2011). Fundamentos de marketing: entorno, consumidor, estrategia e investigación comercial. Recuperado de http://ebookcentral. proquest.com

[3] David, F., (2013). Conceptos de Administración estratégica, México: Editorial Pearson.

[4] Euromonitor International , (2017). Beer in Perú. Recuperado de: http://www.portal. euromonitor.com

[5] Fernández, A., (2004). Dirección y planificación estratégica en empresas y organizaciones. Recuperado de: http://ebookcentral.proquest. com

[6] Flores, R., (2013). Observando observadores: Una Introducción a las Técnicas Cualitativas de Investigación Social. Santiago: Universidad Católica de Chile.

[7] Giraldo, M., y David, J. E. (Eds.). (2016). Gerencia de marketing. Recuperado de http:// ebookcentral.proquest.com

[8] Grande, I. y Abascal E., (2009). Fundamentos y técnicas de investigación comercial. Madrid: Editorial ESIC.

[9] Kotler, P. y Armstrong, G.(2013). Fundamentos de Marketing. México: Editorial Pearson.

[10] Malhotra, N., (2008). Investigación de Mercados. México: Editorial Pearson.

[11] Palacios, L., (2009). Dirección estratégica. Recuperado de http://ebookcentral.proquest. com

[12] Oficina de estudios económicos (OEE). Ministerio de la Producción. Estadística de la Micro, Pequeña y Mediana Empresa (MIPYME). Recuperado de: http:// demi.produce.gob.pe/ estadistica/mypime

[13] Reyes, A., (2005). Fabricación artesanal de cerveza. Recuperado de: http://www.ellibrototal. com/ltotal/?t=1\&d=252_295_1_1_252

[14] Simonazzi, A. (2009). Cerveza. Recuperado de http://ebookcentral.proquest.com

[15] Villanueva, J. y De Toro, J. (2017). Marketing estratégico. Recuperado de: http:// ebookcentral.proquest.com

[16] Yuni, J. y Urbano, C., (2006), Técnicas para investigar y formular proyectos de investigación 2, Córdova, Argentina: Editorial Brujas. 